\title{
New Onset Refractory Status Epilepticus in an Adult Patient with Autoimmune Encephalitis Responding to Vitamin B6 Pyridoxine
}

\author{
Khaleel Al Shaikhli and Ahmed Sameer Nadeem* \\ Department of Neurology, Khoula Hospital, MOH Muscat-Oman
}

Submission: May 08, 2020; Published: May 21, 2020

*Corresponding author: Ahmed Sameer Nadeem, Department of Neurology-Khoula Hospital, MOH Muscat-Oman

Summary

New onset refractory status epilepticus is a difficult to treat neurological emergency. We report here a 39 years old male admitted to our hospital with new onset seizures presented as status epilepticus. Clinical evaluation and extensive investigations revealed an underlying autoimmune-mediated etiology with positivity for anti-NMDAR autoimmune encephalitis. His seizures could not be controlled, and he remained in a super-refractory status epilepticus, despite treatment with multiple anti-epileptic drugs in their proper doses, use of IV anesthetics in addition to a high dose corticosteroid and IVIG courses for treating his underlying disease. The patient had no previous significant past medical history, no family history of a seizure disorder and no comorbidities. Remarkably, the patient's seizures responded successfully to treatment with vitamin B6 (Pyridoxine). He was discharged, after 117 days staying in hospital, with almost complete neurologic recovery and without recurrence of seizures after achieving control with Pyridoxine.

We highlight here the possible role of Pyridoxine to terminate refractory seizures in patients with autoimmune encephalitis and to ensure neuroprotection, while the underlying etiology addressed with immune-modulating therapy. Moreover, we encourage clinicians to consider Pyridoxine deficiency as a potential etiology of new onset refractory status epilepticus and seizures, even in adult patients who suffer from other underlying diseases which can cause seizures.

Keywords: New onset seizure;Refractory status epilepticus; Autoimmune encephalitis;Anti-NMDAR; Pyridoxine

\section{Introduction}

Status Epilepticus (SE) is a medical neuro-emergency describes persistent or recurring seizures without a return to baseline mental status and associated with significant morbidity and mortality[1]. Although the great majority of SE patients have an underlying brain condition causing their status seizure (such as brain tumor, brain infection, brain trauma, or stroke), it can occur in the context of epilepsy, usually precipitated by drug withdrawal, inter-current illness or metabolic disturbance, or by the progression of the underlying disease[1,2]. Around $10 \%$ of epileptic patients may present with SE as a first seizure[2].It is critically important to rapidly institute care that simultaneously stabilizes the patient medically, identifies and manages any precipitating conditions, and terminates seizures. Seizure management involves emergent treatment with benzodiazepines followed by urgent therapy with adequate doses of anti-seizure medications. If seizures persist, then Refractory Status Epilepticus (RSE) is diagnosed and the management options include additional anti-seizure medications or infusions of Midazolam or pentobarbital [3]. Definitions for RSE have varied in seizure durations (no time criteria, 30 minutes, one hour, or two hours) and/or lack of response to different numbers (two or three) and types of anticonvulsants, however, the Neurocritical Care Society guideline states that patients who continue to experience either clinical or electrographic" seizures after receiving adequate doses of an initial benzodiazepine followed by a second acceptable anticonvulsant will be considered refractory and the guidelines recommend rapid advancement to "pharmacologic coma induction rather than sequential trials of many urgent control anticonvulsants[3,4].

In some patients, RSE may last for weeks to months, despite 
treatment with adequate levels of multiple anticonvulsants medications. This lengthy course RSE is referred to as malignant RSE [4] or super-refractory SE (SRSE) [5,6]. Malignant RSE is usually associated with an autoimmune, infectious or inflammatory etiology, younger age patients, previous good health, and it carries high rates of morbidity and mortality $[4,7,8]$.New onset RSE (NORSE) is a rare but challenging condition, characterized by the occurrence of a prolonged period of refractory seizures with no readily identifiable cause in otherwise healthy individuals [9] Although it is reported that autoimmune encephalitis is the most commonly identified cause of new onset refractory status epilepticus, around half of the patients remain cryptogenic [911]. A well-known cause of intractable and refractory seizures is Pyridoxine (vitamin B6) deficiency; however, most of the reported cases of this condition are in infants and neonates[12,13].This article reports a case of a young man who suffered from new onset RSE, diagnosed to have anti-NMDAR encephalitis and his seizures were successfully treated with vitamin B6.

\section{The Case Report}

A 39 years old expatriate male, presented with 3 days history of dizziness, fatigue, mild fever and slight behavior changed. The patient started to have repeated attacks of generalized tonicclonic fits, with up rolling eyes, foaming and without regaining consciousness between these attacks. He was brought to the emergency department at our hospital, with a state of generalized tonic-clonic seizures, where he received diazepam $10 \mathrm{mg}$ IV, which was repeated after 20 minutes because of persistent fitting; in addition to $1000 \mathrm{mg}$ IV Phenytoin as well as to Midazolam $(0.2 \mathrm{mg} / \mathrm{kg}$ IV bolus followed by $0.5 / \mathrm{kg} / \mathrm{hr}$ infusion), but the clinical fits further persist, therefore, the patient was shifted to the ICU, intubated for airway and neurologic protection, Phenobarbitone IV $200 \mathrm{mg}$ at a rate of $100 \mathrm{mg} / \mathrm{min}$ was given and sedation with Midazolam infusion with up-titrated to $20 \mathrm{mg} / \mathrm{h}$.

Electroencephalography (EEG) was done at admission in the emergency department and showed initially status patterns of continuous generalized flattening of the normal background rhythms, followed by repeated spells of generalized low voltage fast activity and polyspikes that increase in amplitude and decrease in frequency until becoming obscured by muscle and movement artifacts. These patterns change, as the seizure clinically moves into the clonic phase, to a checkerboard type of muscle artifacts corresponding to the rhythmic jerking movements observed clinically (all four limbs and right-side facial twitching). The EEG further shows diffuse suppression of cerebral activity during breaks between seizures. Subsequently, in the ICU and after sedation, continuous electroencephalogram monitoring showed breakthrough seizures, in spite of pentobarbital-induced burst suppression, in the forms of frequent generalized spikes, polyspikes and wave discharges (predominantly over the left hemisphere) and generalized Periodic Lateralized Epileptiform Discharges (PLEDs) synchronizing with the jerking movements of the patient.
Seizures treatment was initiated with Anti-Epileptic Drugs (AEDs) regimen of Levetiracetam and Valproic acid added to the already in use Phenobarbitone and Phenytoin; however, events of facial twitching and limbs' convulsions were observed clinically and epileptic discharges activity remained evident on EEG. After 10 days of intubation, the patient underwent a tracheostomy procedure due to prolonged mechanical ventilation requirements.

The patient was clinically evaluated and extensively investigated. Given the suspension of meningoencephalitis, from an infectious etiology by history and presentation, the patient was treated empirically with vancomycin, ceftriaxone, and acyclovir. However, Lumbar puncture was done and CSF analysis showed a clear CSF with pleocytosis, WBC cell count of 64 (normal 0) , with $90 \%$ lymphocytes and only 2 RBCs, elevated protein $94 \mathrm{mg} /$ $\mathrm{dl}$ (normal 25-45mg/dl) and normal glucose level $4.43 \mathrm{mmol} / \mathrm{L} \mathrm{(}$ blood glucose $6.2 \mathrm{mmol} / \mathrm{L}$ ). Indian-ink stain test for Cryptococcus and quantiferon test for TB were negative; in addition, the CSF culture and viral PCR testing (for HSV, adenovirus, enterovirus, Varicella and Mumps) were all negative. Based on the results of lumbar puncture and cerebral spinal fluid analysis, acyclovir and antibiotics were discontinued.The patient's blood tests showed hemoglobin level of $11.4 \mathrm{gm} / \mathrm{dl}$, total WBC count of 19,900/ $\mathrm{cmm}$, platelets were normal $345,000 / \mathrm{cmm}$ and normal rest of CBC indices. C-reactive protein test (CRP) was high $267.6 \mathrm{mg} / \mathrm{L}$ (normal $0-5 \mathrm{mg} / \mathrm{L}$ ), normal random blood sugar (RBS), Liver and renal function tests (LFT \& RFT) and normal Thyroid-Stimulating Hormone (TSH) level. Serum electrolytes all were normal except serum magnesium which was low 0.49 milligrams per deciliter (normal 0.66-1.07 milligrams per deciliter;) so oral supplements was given and the level became within normal (0.72 milligrams per deciliter). The patient's Blood cultures were negative and tests for HIV 1, 2 as well as Elisa test for syphilis.Subsequently, MRI brain was done and showed variably distributed parenchymal T2/FLAIR hyperintensities in the hyppocampal region, para hippocampus gyrus and in the amygdale with subtle involvement of the right insular cortex and bilateral medial temporal cortical swelling, consistent with encephalitis. All lesions were not enhancing with Gadolinium contrast.

With the suspicion of autoimmune encephalitis, from the clinical history, CSF and MRI findings, CSF was sent for anti-Nmethyl-D-aspartate receptor (NMDAR) antibodies and the results came positive confirming the diagnosis of autoimmune antiNMDAR encephalitis. No oligoclonal bands were detected in the CSF.

Further investigations, including paraneoplastic and vasculitis panels, were done and came to be with negative results. He was also investigated with pelvic ultrasonography, CT scan for chest, abdomen and pelvis which excluded teratoma and other tumors. In addition, his serum tests for Antiphospholipids syndrome, Lupus anticoagulant, Limbic encephalitis screen and antiNeuronal antibodies were all negative. 


\section{Open Access Journal of Neurology \& Neurosurgery}

Based on the diagnosis, the patient received two courses of Methylprednisolone IV infusion 1gm/ day for 5 days, two weeks apart, yet the seizures continued. Therefore, IVIG course of $2.5 \mathrm{gm} / \mathrm{kg}$ divided to 5 days was also given. However, the patient continued to have generalized seizures and a Propofol infusion started at $2 \mathrm{mg} / \mathrm{kg} / \mathrm{h}$ up titrated and with intermittent boluses to achieve and maintain burst suppression on EEG.

During the following days, maximum doses of these AEDs were reached (Phenobarbitone dose was escalated up to $500 \mathrm{mg} /$ day IV, Phenytoin given $1000 \mathrm{mg}$ to start then adjusted $400 \mathrm{mg}$ IV daily according to its' serum level, Valproic acid $400 \mathrm{mg}$ bid IV then escalated up to $1000 \mathrm{mg}$ bid, and Levetiracetam given as $1500 \mathrm{mg}$ bid then raised to $2000 \mathrm{mg}$ bid) and an addition of Lamotrigine ,up to $250 \mathrm{mg}$ bid, then Topiramate, which was slowly increased up to $200 \mathrm{mg}$ bid, were also tried. However, the patient continued to experience breakthrough seizures, evident on EEG and on clinical exam as facial twitching and rhythmic limbs' movement whenever sedation was decreased, consistent with SRSE. Thus, Ketamine IV, in a loading dose of $1.5 \mathrm{mg} / \mathrm{kg}$, followed by 2.75 $\mathrm{mg} / \mathrm{kg} / \mathrm{h}$ continuous infusion, was subsequently added. Plasma exchange (plasmapheresis) therapy was also offered but the patient's sponsor deferred the treatment.

On day 68 in the ICU, a trial of Pyridoxine 50mg tid, orally through nasogastric tube, was given. After 30 hours, the patient's clinical seizures had resolved, and the EEG ictal discharges ceased. Throughout the rest of the admission, the patient was gradually and successfully extubated and weaned off from the ventilator, we slowly tapered the Phenytoin, then the Phenobarbitone and the patient had no more fits.

A challenge test was done by stopping the Pyridoxine, after 20 days, fits recurred after 24 hours as complex partial motor seizures (focal motor seizures with impaired awareness) affecting the face and the right arm with loss of consciousness, so he was reintubated and ventilated, and Pyridoxine restarted again. Seizures subsided after 12 hours and the patient was off ventilator then extubated after 2 days.Our patient started to recover clinically and was able to tolerate eating meals by mouth, started to walk alone with some difficulty and started a physiotherapy and rehabilitation program to return to his baseline functional status. Most AEDs were tapered and stopped gradually; he was discharged, after spending a total of 117 days in hospital, on Levetiracetam (1500 mg bid) in a good condition. We discussed with the patient the high risk of relapse in regard to his autoimmune anti-NMDAR encephalitis and the need for Rituximab treatment, but he was reluctant for a 6 months cycle of therapy and wanted to travel back home. However, we kept him under frequent follow ups, and he was stable without any relapse.

\section{Discussion}

New-onset Refractory Status Epilepticus (NORSE) is a lifethreatening neurological emergency which happens when an otherwise healthy patient presents with SE, the cause of SE cannot be identified initially and when SE cannot be controlled with the standard anti-seizure medicine (refractory) [9,14]. Although some cases with NORSE have been reported to recover completely [11-15], it can cause significant brain damage and it is reported that between $20-40 \%$ of people do not survive [1416]. Lifelong epilepsy, as well as physical and mental disabilities were also reported in most cases survivors [11,14]; therefore, treatment of NORSE should be initiated rapidly and effectively to terminate seizures and to target the underlying cause if found. More extensive testing should be performed if the clinical findings and the initial investigations fail to find an underlying cause. Tests have to be performed to rule out other known causes of new-onset refractory SE, such as inflammatory and autoimmune diseases, genetic disorders, metabolic disorders, and less common viral infections $[11,17,18]$.

Autoimmune encephalitis, either nonparaneoplastic or paraneoplastic, was the most common reported cause of NORSE [4,19-22] and anti-NMDAR encephalitis cases presenting as NORSE are reported $[23,24]$ knowing that most published cases of NORSE predate the discovery of anti-NMDAR antibodies[11,25].Here, we present a case of NORSE with underlying nonparaneoplastic autoimmune encephalitis etiology (diagnosed by elevated protein in the CSF with pleocytosis, positive CSF anti-NMDAR antibodies and by brain MRI findings) in a previously healthy young male. Although general expert consensus in the literature recommends approaching NORSE with pharmacologic-induced coma and continuous infusion of IV anesthetic agents, to suppress brain activity and preserve normal brain physiology [26], our patient did not respond and his NORSE became super-refractory. He continued seizing despite the use of all the previous management; in addition to benzodiazepines, various AEDs and the treatment with Methylprednisolone and IVIG for his autoimmune encephalitis. Our patient's seizures stopped, and he ultimately made almost complete neurological recovery after administration of vitamin B6 (Pyridoxine).

Pyridoxine-dependent seizures are usually related to a rare autosomal recessive mutation in the ALDH7A1 gene; therefore it is generally considered in neonates with seizures, although there are also reports of older patients including infants, children and even few adults with SE controlled by Pyridoxine[27-30]. The diagnosis of pyridoxine-responsive seizures is made when administration of Pyridoxine (usually, intravenous 100mg given for one to five doses) terminates seizures, typically within hours of administration [31]. Moreover, Pyridoxine (vitamin B6) deficiency is a well-known cause of refractory SE; however, most of the reported cases were in infants and neonates [12,13]. Few adult cases of pyridoxine deficiency related RSE seizures have been reported in the literature[32,33] and the majority were associated with nutritional deficiencies and comorbidities [34-37] or with pregnancy [38].Vitamin B6 deficiency in adults is rare and may result from dietary deficiency (especially in elderly and alcoholics), and in patients with liver disease, chronic kidney 
disease on dialysis, rheumatoid arthritis, women with type 1 diabetes, as well as during pregnancy and in those patients with HIV who develop an increased risk of vitamin B6 deficiency, despite adequate dietary intakes $[26,39,40]$. Certain medications can also affect the availability of vitamin B6 in the body or interfere with its' metabolism, such as anticonvulsants, corticosteroids, Isoniazid, Cycloserine, and Penicillamine [41].

In this particular case of NORSE, the patient is a young, completely healthy male and without history of alcohol or any medication intake which makes this case unique and worth reporting as his seizures were only stopped by Pyridoxine, despite the extensive treatment and prolonged ICU admission. Although the underlying etiology was autoimmune-mediated epilepsy, the addition of vitamin B6 achieved an excellent outcome leading to almost complete neurological recovery after.

\section{Conclusion}

New onset refractory status epilepticus is a serious condition that needs ICU, intubation, ventilation and trial of medications to stop the fits. The finding that many cases of NORSE are of autoimmune origin suggests that these etiologies should be aggressively sought.The unusual outcome demonstrated in this case report raises a discussion of the optimal approach and treatment as applied to a case of NORSE, where rapid and almost complete neurological recovery was achieved with the additional trial of Pyridoxine.We recommend that a trial of Pyridoxine to be administrated in cases of intractable/refractory status seizures and it can be included in the status epilepticus treatment protocols of adult patients with refractory status, regardless the underlying cause. In addition, the diagnosis of late onset Pyridoxinedependent epilepsy should be considered especially in countries where consanguineous marriage is often seen.

\section{Acknowledgment}

We would like to express our sincere gratitude and appreciation to the ICU and Emergency departments at Khoula hospital for their valuable contribution that greatly assisted the completion of this case report.

\section{Consent}

Informed consent for the publication of the clinical details, tests' results and the radiological images was obtained from the patient and documented in the patient's electronic file.

\section{Competing Interest}

The authors declare no financial competing interest, as well as non-financial competing interests (personal, ideological, academic, intellectual, commercial or any other) in relation.

\section{References}

1. Al-Mufti F, Claassen J (2014) Neurocritical Care: Status Epilepticus Review. Critical Care Clinics 30(4): 751-764.
2. Trinka E, Höfler J, Zerbs A (2013) Causes of status epilepticus. Epilepsia 53 Suppl 4: 127-138.

3. Brophy GM, Bell R, Claassen J, Brian Alldredge, Thomas P Bleck, et al. (2012) Guidelines for the evaluation and management of status epilepticus. Neurocrit Care 17(1): 3-23.

4. Holtkamp M, Othman J, Buchheim K, Masuhr F, Schielke E, et al. (2005) A Malignant variant of status epilepticus. Arch Neurol 62(9):14281431.

5. Shorvon S, Ferlisi M (2011) The treatment of super-refractory status epilepticus: a critical review of available therapies and a clinical treatment protocol. Brain 134: 2802-2918.

6. Shorvon S (2011) Super-refractory status epilepticus: An approach to therapy in this difficult clinical situation. Epilepsia 52 (Suppl 8): 53-56.

7. Wilder-Smith EP, Lim EC, Teoh HL, VK Sharma, JJ H Tan, et al. (2005) The NORSE (new-onset refractory status epilepticus) syndrome: defining a disease entity. Ann Acad Med Singapore 34: 417-420.

8. Van Lierde I, Van Paesschen W, Dupont P, Maes A, Sciot R (2003) De novo cryptogenic refractory multifocal febrile status epilepticus in the young adult: a review of six cases. Acta Neurol Belg 103(2): 88-94.

9. Costello DJ, Kilbride RD, Cole AJ (2009) Cryptogenic new onset refractory status epilepticus (NORSE) in adults: infectious or not? J Neurol Sci 277: 26-31.

10. Teneille E Gofton, Nicolas Gaspard, Sara E Hocker, Tobias Loddenkemper, Lawrence J Hirsch (2019) New onset refractory status epilepticus research, what is on the horizon? Neurology 92 (17): 802 810.

11. Gaspard N, Foreman BP, Alvarez V, Christian Cabrera Kang, John C Probasco, et al. (2015) New-onset refractory status epilepticus: Etiology, clinical features, and outcome. Neurology 85(18): 1604-1613.

12. Hunt AD Jr, Stokes JJr, McCrory WW, Stroud HH (1954) Pyridoxine dependency: Report of a case of intractable convulsions in an infant controlled by pyridoxine. Pediatrics 13(2): 140-145.

13. Baxter P (2001) Pyridoxine dependent and pyridoxine responsive seizures. In: Baxter P, ed. Vitamin Responsive Conditions in Paediatric Neurology. London: MacKeith Press 109-165.

14.Gaspard N New-Onset Refractory Status Epilepticus (NORSE) and Febrile Infection-Related Epilepsy Syndrome (FIRES). National Organization for Rare Disorders (NORD).

15. Gaspard N, Hirsch LJ, Sculier C (2018) New-onset refractory status epilepticus (NORSE) and febrile infection-related epilepsy syndrome (FIRES): State of the art and perspectives. Epilepsia 59(4): 745-752.

16. Lowenstein DH, Alldredge BK (1998) Status Epilepticus 338: 970-976.

17. Abend NS, Bearden D, Helbig I, Jennifer McGuire, Sona Narula, et al. (2014) Status epilepticus and refractory status epilepticus management. Semin Pediatr Neurol 21(4): 263-274.

18. Hirsch LJ, Gaspard N, van Baalen A, Rima Nabbout, Sophie Demeret, et al. (2018) Proposed consensus definitions for new-onset refractory status epilepticus (NORSE), febrile infection-related epilepsy syndrome (FIRES), and related conditions. Epilepsia 59(4): 739-744.

19. Holtkamp M, Othman J, Buchheim K, Meierkord H (2005) Predictors and prognosis of refractory status epilepticus treated in a neurological intensive care unit. J Neurol Neurosurg Psychiatry 76(4): 534-539.

20. Glaser CA, Gilliam S, Honarmand S, Jay H Tureen, Daniel H Lowenstein, et al. (2008) Refractory status epilepticus in suspect encephalitis. Neurocrit Care 9(1): 74-82. 


\section{Open Access Journal of Neurology \& Neurosurgery}

21. Thakur KT, Motta M, Asemota AO, David R Benavides, Eric B Schneider, et al. (2013) Predictors of outcome in acute encephalitis. Neurology 81(9): 793-800.

22. Singh TD, Fugate JE, Rabinstein AA (2015) The spectrum of acute encephalitis: causes, management, and predictors of outcome. Neurology 84(4): 359-366.

23. Legault C, Lapointe S, Giacomini P, Teitelbaum J, La Piana R (2015) NMDA-Receptor Encephalitis: An Unsual Case of Refractory Status Epilepticus Neurology 84 (14 Supplement) P6: 286.

24. Liu B, Zhou Y, Meng L, Skinner H (2018) A Survival Case of Superrefractory Status Epilepticus due to Glutamic Acid Decarboxylase Antibodies-associated Limbic Encephalitis. Cureus 10(8): e3125.

25. Dalmau J, Tüzün E, Wu HY, Jaime Masjuan, Jeffrey E Rossi, et al. (2007) Paraneoplastic anti-Nmethyl-D-aspartate receptor encephalitis associated with ovarian teratoma. Ann Neurol 61(1): 25-36.

26. Hocker SE (2015) Status Epilepticus. Neurocrit Care 21: 1362-83.

27. Yoshii A, Takeoka M, Kelly PJ, Krishnamoorthy KS (2005) Focal status epilepticus as atypical presentation of pyridoxine-dependent epilepsy. J Child Neurol 20: 696-698.

28. Goutieres F, Aicardi J (1985) Atypical presentations of pyridoxinedependent seizures: a treatable cause of intractable epilepsy in infants. Ann Neurol 17(2): 117-120.

29. Chou ML, Wang HS, Hung PC, Sun PC, Huang SC (1995) Late-onset pyridoxine-dependent seizures: report of two cases. Acta Paediatr Taiwanica 36(6): 434-437.

30. Kluger G, Blank R, Paul K, E Jansen, C Jakobs, et al. (2008) Pyridoxine dependent epilepsy: normal outcome in a patient with late diagnosis after prolonged status epilepticus causing cortical blindness. Neuropediatrics 39(5): 276-279.

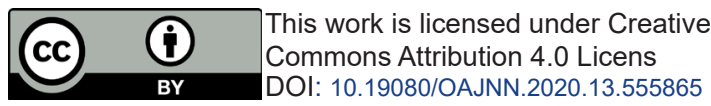

31. Russell KE, Mulligan SR, Mallory LA (2012) Diagnosis of pyridoxinedependent seizures in a nineteen-year-old patient. Pediatr Neurol 47(2):141-143

32. Tajender V, Saluja J (2006) INH induced status epilepticus: Response to pyridoxine. Indian J Chest Dis Allied Sci 48(3): 205-206.

33. Gerlach AT, Thomas S, Stawicki SP, Whitmill ML, Steinberg SM, et al. (2011) Vitamin B6 deficiency: A potential cause of refractory seizures in adults. J Parenter Enter Nutr 35(2): 272-275.

34. Asnis DS, Bhat JG, Melchert AF (1993) Reversible seizures and mental status changes in a dialysis patient on isoniazid preventive therapy. Ann Pharmacother 27(4): 444-446.

35. Skodda S, Müller T (2013) Refractory epileptic seizures due to vitamin B6 deficiency in a patient with Parkinson's disease under duodopa therapy. J Neural Transm 120(2): 315-318.

36. Tong Y (2014) Seizures caused by pyridoxine (vitamin B6) deficiency in adults: A case report and literature review. Intractable Rare Dis Res 3(2): 52-56.

37. Lee DG, Lee Y, Shin H, Kyusik Kang, Jong-Moo Park, et al. (2015) Seizures Related to Vitamin B6 Deficiency in Adults. J Epilepsy Res 5(1): 23-24.

38. Schulze-Bonhage A, Kurthen M, Walger P, Elger CE (2004) Pharmacorefractory status epilepticus due to low vitamin B6 levels during pregnancy. Epilepsia 45(1): 81-84.

39. Bowman BA, Russell RM (2006) Present Knowledge in Nutrition ( $9^{\text {th }}$ Ed). Washington, DC: ILSI Press, 273.

40. Rall LC, Meydani SN (1993) Vitamin B6 and immune competence. Nutrition Reviews 51(8): 217-225.

41. Bhagavan HN (1985) Interraction between vitamin B6 and drugs. In Reynolds RD, Leklem JE. Vitamin B6: Its role in health and disease. New York: Liss : 401-415.

\section{Your next submission with Juniper Publishers} will reach you the below assets

- Quality Editorial service

- Swift Peer Review

- Reprints availability

- E-prints Service

- Manuscript Podcast for convenient understanding

- Global attainment for your research

- Manuscript accessibility in different formats

( Pdf, E-pub, Full Text, Audio)

- Unceasing customer service

Track the below URL for one-step submission

https://juniperpublishers.com/online-submission.php 